\title{
Contribution à la connaissance des abeilles de la Loire-Atlantique, du Maine-et-Loire et de la Vendée
}

\author{
par Violette Le FÉON*, Doriane BLotTIÈRE**,***, David GenOud**** \& Olivier LAMBeRT** \\ Publication : novembre 2020
}

\begin{abstract}
Résumé. Nous présentons une synthèse des résultats obtenus par le Centre Vétérinaire de la Faune Sauvage et des Écosystèmes (CVFSE, Nantes, France) au cours de ses inventaires d'abeilles entre 2014 et 2018. Les collectes ont été réalisées au filet sur 84 sites répartis dans 43 communes des Pays de la Loire (32 en Loire-Atlantique, 4 en Maine-et-Loire et 7 en Vendée). Les milieux prospectés étaient très divers : milieux urbains, zones agricoles, espaces naturels prairiaux, forestiers ou littoraux. Cette étude a permis d'approfondir les connaissances sur la distribution de 278 espèces d'abeilles, parmi lesquelles plusieurs étaient jusqu'alors inconnues du Massif armoricain (Andrena florivaga, Chelostoma distinctum, Coelioxys obtusa, Eucera clypeata, E. pannonica, Hylaeus clypearis, H. leptocephalus, H. punctatus, Lasioglossum tarsatum, Nomada errans, $N$. furva, N. lisenmaieri, $N$. rubiginosa, $N$. villosa et Sphecodes pseudofasciatus). Elle a mis en évidence la richesse des milieux naturels, en particulier les habitats littoraux dunaires, mais aussi l'intérêt de certaines zones urbaines ou péri-urbaines, pour peu que la gestion des espaces soit compatible avec la présence des abeilles.
\end{abstract}

Mots-clés. Abeilles, inventaire, milieux anthropisés, milieux naturels, Massif armoricain.

Abstract. Contribution to the knowledge of bees from Loire-Atlantique, Maine-et-Loire and Vendée. We present an overview of a study on bees carried out by the Centre Vétérinaire de la Faune Sauvage et des Écosystèmes (CVFSE, Nantes, France) between 2014 and 2018. Bees were collected through net sampling in 84 sites located in 43 cities of the French region Pays de la Loire (32 cities in Loire-Atlantique, 4 in Maine-et-Loire, and 7 in Vendée). Sampling occurred in different types of habitats: urban areas, agricultural areas, and semi-natural habitats like grasslands, woodlands, and coastal zones. This study provided information on the distribution of 278 bee species, among which several were previously unknown to the Armorican Massif (Andrena florivaga, Chelostoma distinctum, Coelioxys obtusa, Eucera clypeata, E. pannonica, Hylaeus clypearis, H. leptocephalus, H. punctatus, Lasioglossum tarsatum, Nomada errans, $N$. furva, $N$. lisenmaieri, $N$. rubiginosa, $N$. villosa, and Sphecodes pseudofasciatus). It highlighted the great bee species richness in semi-natural habitats, notably in the coastal dunes, but also in some urban or suburban sites, as long as the management is favourable to bees.

Keywords. Bees, species list, anthropogenic habitats, natural habitats, Armorican Massif.

\section{Introduction}

Dans la région des Pays de la Loire, plusieurs travaux sur les abeilles ont été menés depuis le début des années 2000, en particulier la réalisation d'un atlas des bourdons de Loire-

\footnotetext{
* Écologue, 10 rue de l'Olivraie, F-44200 Nantes violette.lefeon@gmail.com

** Centre Vétérinaire de la Faune Sauvage et des Écosystèmes (CVFSE) des Pays de la Loire Oniris, École Nationale Vétérinaire, Agroalimentaire et de l'Alimentation Nantes Atlantique Site de la Chantrerie, route de Gachet, CS 40706, F-44307 Nantes cedex 03 olivier.lambert@oniris-nantes.fr

*** Actuellement au MNHN, UMS PatriNat (OFB - CNRS - MNHN), 43 rue Buffon, CP135, F-75005 Paris - doriane.blottiere@mnhn.fr

**** Écologue, 2 ter avenue des Roses, F-87240 Ambazac . dge-davidgenoud@orange.fr
}

Atlantique (MAHÉ 2015) et des inventaires ponctuels (LACHAUD \& MAHÉ 2008 ; MAHÉ 2009 ; HERBRECHT 2012, 2014 ; HERBRECHT \& LAIR 2012a, 2012b, 2013), principalement localisés sur le littoral de la Loire-Atlantique. De 2014 à 2018, plusieurs inventaires des abeilles ont été réalisés par le Centre Vétérinaire de la Faune Sauvage et des Écosystèmes des Pays de la Loire (CVFSE) dans les départements de la Loire-Atlantique (44), du Maine-et-Loire (49) et de la Vendée (85) dans le cadre de différents programmes de recherche ou d'amélioration des connaissances naturalistes. Nous présentons ici une synthèse des informations apportées par ces collectes réalisées 


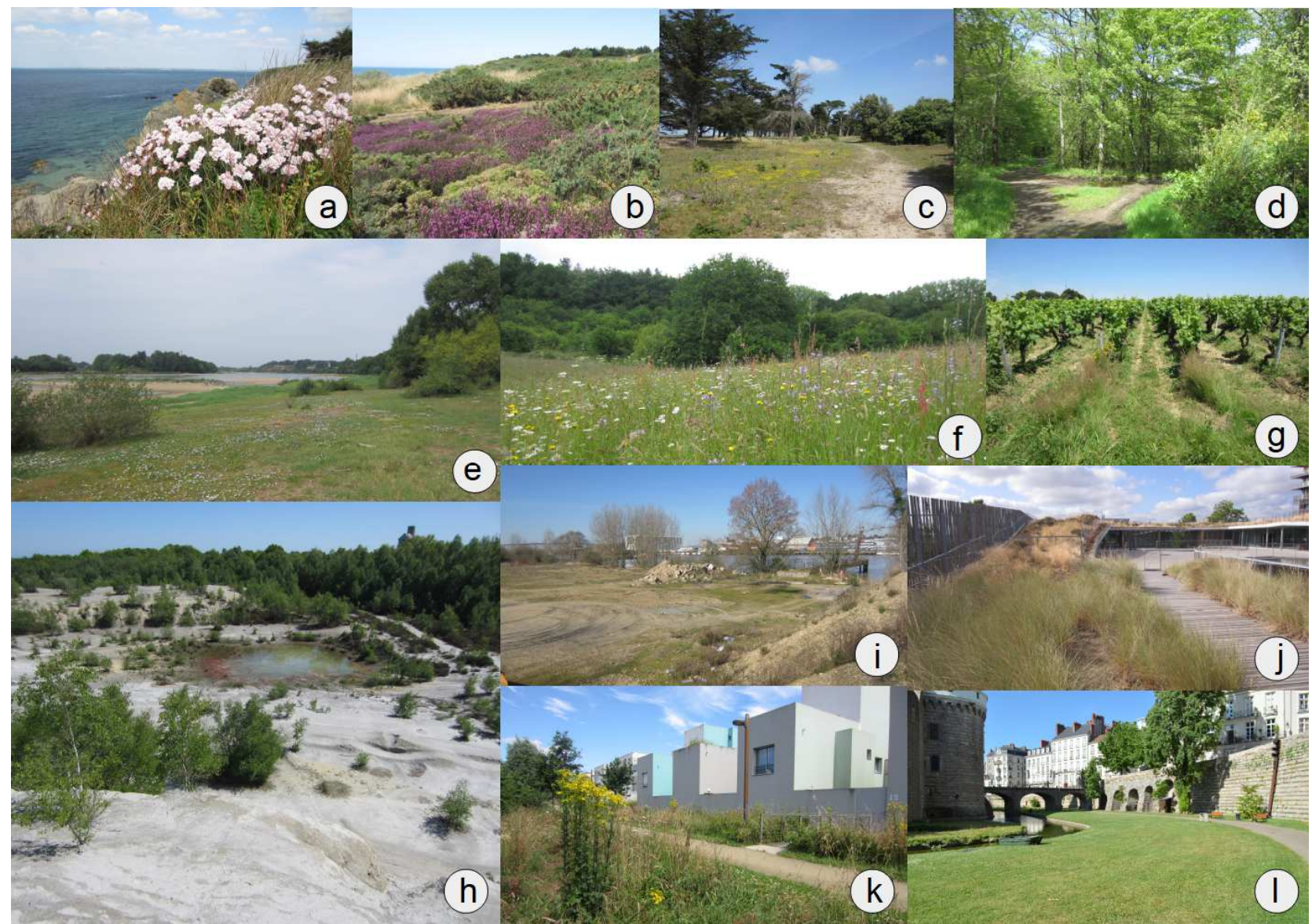

Figure 1. Diversité des milieux échantillonnés : (a) Rio More (Piriac-sur-Mer, 44). (b) Port aux Goths (Préfailles, 44). (c) Dune du Collet (Les Moutiers-en-Retz, 44). (d) Forêt de Touffou (Vertou, 44). (e) Île du Bernardeau (Ancenis, 44). (f) Vallon des Saules (La Chapelle-sur-Erdre, 44). (g) Vigne en agriculture biologique (Saint-Léger-les-Vignes, 44). (h) Terril (Abbaretz, 44). (i) Sablière de Trentemoult (Rezé, 44). (j) Toit végétalisé de l'école Aimé Césaire (Nantes, 44). (k) Quartier Bottière-Chénaie (Nantes, 44). (I) Douves du Château des Ducs de Bretagne (Nantes, 44). Photos Olivier LAMBERT.

dans des milieux très divers (figure 1) : milieux urbains, zones agricoles, espaces naturels prairiaux, forestiers ou littoraux.

\section{Matériel et Méthodes}

Les collectes ont été réalisées dans 43 communes (32 en Loire-Atlantique, 4 en Maine-et-Loire et 7 en Vendée) (figure 2). Dans la majorité des cas (27 communes), un unique site a été échantillonné durant une seule année (tableau I). Pour les autres communes, un même site a été échantillonné durant plusieurs années ou plusieurs sites ont été échantillonnés durant une ou plusieurs années. $\mathrm{Au}$ total, 84 sites ont été échantillonnés. L'effort d'échantillonnage maximal concerne Nantes, commune pour laquelle on dénombre 12 sites étudiés.

Sur chaque site, pour une année donnée, les collectes ont été réalisées une fois par mois de mars-avril à août-septembre (soit cinq ou six sessions de collecte dans la majorité des cas). Font exception les îles ligériennes d'Ancenis (44) : l'île du Bernardeau a été échantillonnée uniquement les 5 juin et 3 juillet 2015 tandis que l'île aux Moines et l'île Kerguelen ne l'ont été que le 27 juin 2018.

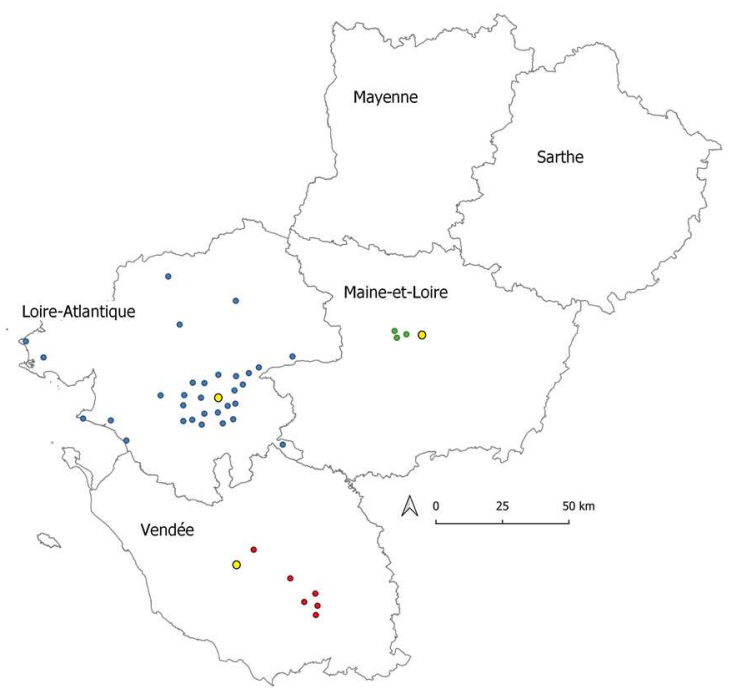

Figure 2. Localisation des 43 communes de l'étude au sein de la région des Pays de la Loire. Les préfectures Nantes, Angers et La Roche-sur-Yon sont représentées par des points jaunes. 
Tableau I. Nombre de spécimens et d'espèces (richesse spécifique) collectés dans les 43 communes de l'étude. Les couleurs renseignent sur l'effort d'échantillonnage appliqué dans chaque commune et informent donc sur le niveau de connaissance : bleu = un site une année avec 3 ou 4 sessions de collecte ; vert clair $=$ un site une année avec plus de 4 sessions ; vert foncé $=2$ sites une année, soit une douzaine de sessions au total ; orange $=2$ ou 3 sites deux années ou 5 sites une année, entre 23 et 37 sessions au total ; rouge $=8$ sites échantillonnés chacun une ou deux années pour La Roche-sur-Yon et 12 sites échantillonnés une, deux ou trois années pour Nantes.

\begin{tabular}{|c|c|c|c|}
\hline Département & Commune & Nombre de spécimens & Richesse spécifique \\
\hline \multirow[t]{32}{*}{ Loire-Atlantique } & Abbaretz & 352 & 28 \\
\hline & Ancenis & 220 & 35 \\
\hline & Basse-Goulaine & 182 & 28 \\
\hline & Blain & 109 & 45 \\
\hline & Bouaye & 147 & 40 \\
\hline & Bouguenais & 176 & 23 \\
\hline & Carquefou & 208 & 20 \\
\hline & Couëron & 95 & 36 \\
\hline & Gétigné & 218 & 62 \\
\hline & Guémené-Penfao & 63 & 34 \\
\hline & Guérande & 197 & 58 \\
\hline & La Chapelle-sur-Erdre & 245 & 54 \\
\hline & Le Cellier & 180 & 23 \\
\hline & Le Pellerin & 160 & 31 \\
\hline & Les Moutiers-en-Retz & 834 & 90 \\
\hline & Les Sorinières & 156 & 59 \\
\hline & Mauves-sur-Loire & 614 & 67 \\
\hline & Nantes & 1893 & 134 \\
\hline & Orvault & 185 & 47 \\
\hline & Piriac-sur-Mer & 290 & 37 \\
\hline & Pornic & 401 & 49 \\
\hline & Préfailles & 67 & 28 \\
\hline & Rezé & 468 & 54 \\
\hline & Saint-Aignan-de-Grandlieu & 169 & 26 \\
\hline & Saint-Herblain & 703 & 88 \\
\hline & Saint-Jean-de-Boiseau & 173 & 43 \\
\hline & Saint-Léger-les-Vignes & 142 & 35 \\
\hline & Saint-Sébastien-sur-Loire & 164 & 55 \\
\hline & Sainte-Luce-sur-Loire & 140 & 17 \\
\hline & Sautron & 98 & 32 \\
\hline & Thouaré-sur-Loire & 139 & 43 \\
\hline & Vertou & 588 & 77 \\
\hline \multirow[t]{4}{*}{ Maine-et-Loire } & Angers & 727 & 91 \\
\hline & Beaucouzé & 262 & 59 \\
\hline & Saint-Jean-de-Linières & 51 & 18 \\
\hline & Saint-Lambert-la-Potherie & 200 & 63 \\
\hline \multirow[t]{7}{*}{ Vendée } & Bournezeau & 79 & 17 \\
\hline & La Ferrière & 45 & 11 \\
\hline & La Roche-sur-Yon & 1318 & 120 \\
\hline & Saint-Étienne-de-Brillouet & 51 & 14 \\
\hline & Sainte-Hermine & 62 & 24 \\
\hline & Saint-Juire-Champgillon & 61 & 22 \\
\hline & Thiré & 34 & 12 \\
\hline
\end{tabular}

Toutes les collectes ont été réalisées au filet (type " filet à papillons") lors de sessions d'une heure et demie à trois heures au cours desquelles le site était parcouru à pied par deux personnes formées en amont à la biologie des abeilles. Même si elles peuvent aboutir à un nombre d'espèces détecté moindre que lors de collectes par piégeage (coupelles colorées), les collectes au filet sont considérées comme une méthode performante pour l'inventaire des abeilles (WESTPHAL et al. 2008). 
Tableau II. Liste des espèces d'abeilles collectées entre 2014 et 2018 en Pays de la Loire par le CVFSE (Oniris, Nantes). La dernière colonne indique le statut de chaque espèce dans la liste rouge des abeilles d'Europe (NIETO et al. 2014) : DD = data deficient, LC = least concerned, NT = near threatened, VU = vulnerable, $\mathrm{ND}=$ espèce non documentée dans la liste rouge.

\begin{tabular}{|c|c|c|c|}
\hline Famille & Genre & Espèce & Statut LRE \\
\hline \multirow[t]{52}{*}{ Andrenidae } & Andrena & Andrena agilissima & DD \\
\hline & & Andrena alfkenella & DD \\
\hline & & Andrena ampla & ND \\
\hline & & Andrena angustior & DD \\
\hline & & Andrena apicata & DD \\
\hline & & Andrena argentata & DD \\
\hline & & Andrena barbilabris & DD \\
\hline & & Andrena bicolor & $\mathrm{LC}$ \\
\hline & & Andrena bimaculata & DD \\
\hline & & Andrena carantonica & DD \\
\hline & & Andrena chrysosceles & DD \\
\hline & & Andrena cineraria & $\mathrm{LC}$ \\
\hline & & Andrena cinerea & DD \\
\hline & & Andrena distinguenda & DD \\
\hline & & Andrena dorsata & DD \\
\hline & & Andrena erythrocnemis & DD \\
\hline & & Andrena falsifica & DD \\
\hline & & Andrena ferox & DD \\
\hline & & Andrena flavilabris & DD \\
\hline & & Andrena flavipes & $\mathrm{LC}$ \\
\hline & & Andrena florea & DD \\
\hline & & Andrena florivaga & $\mathrm{LC}$ \\
\hline & & Andrena fulva & DD \\
\hline & & Andrena fulvago & DD \\
\hline & & Andrena fulvata & DD \\
\hline & & Andrena fulvida & NT \\
\hline & & Andrena fuscipes & DD \\
\hline & & Andrena gravida & DD \\
\hline & & Andrena haemorrhoa & $\mathrm{LC}$ \\
\hline & & Andrena helvola & DD \\
\hline & & Andrena humilis & DD \\
\hline & & Andrena labialis & DD \\
\hline & & Andrena labiata & DD \\
\hline & & Andrena lagopus & $\mathrm{LC}$ \\
\hline & & Andrena lathyri & DD \\
\hline & & Andrena livens & $\mathrm{LC}$ \\
\hline & & Andrena minutula & DD \\
\hline & & Andrena minutuloides & DD \\
\hline & & Andrena nigroaenea & $\mathrm{LC}$ \\
\hline & & Andrena nigroolivacea & $\mathrm{LC}$ \\
\hline & & Andrena nitida & $\mathrm{LC}$ \\
\hline & & Andrena nitidiuscula & $\mathrm{LC}$ \\
\hline & & Andrena ovatula & NT \\
\hline & & Andrena pandellei & $\mathrm{LC}$ \\
\hline & & Andrena pilipes & $\mathrm{LC}$ \\
\hline & & Andrena ranunculi & $\mathrm{LC}$ \\
\hline & & Andrena rhenana & DD \\
\hline & & Andrena schencki & DD \\
\hline & & Andrena semilaevis & DD \\
\hline & & Andrena similis & DD \\
\hline & & Andrena simontornyella & $\mathrm{LC}$ \\
\hline & & Andrena strohmella & $\mathrm{LC}$ \\
\hline
\end{tabular}




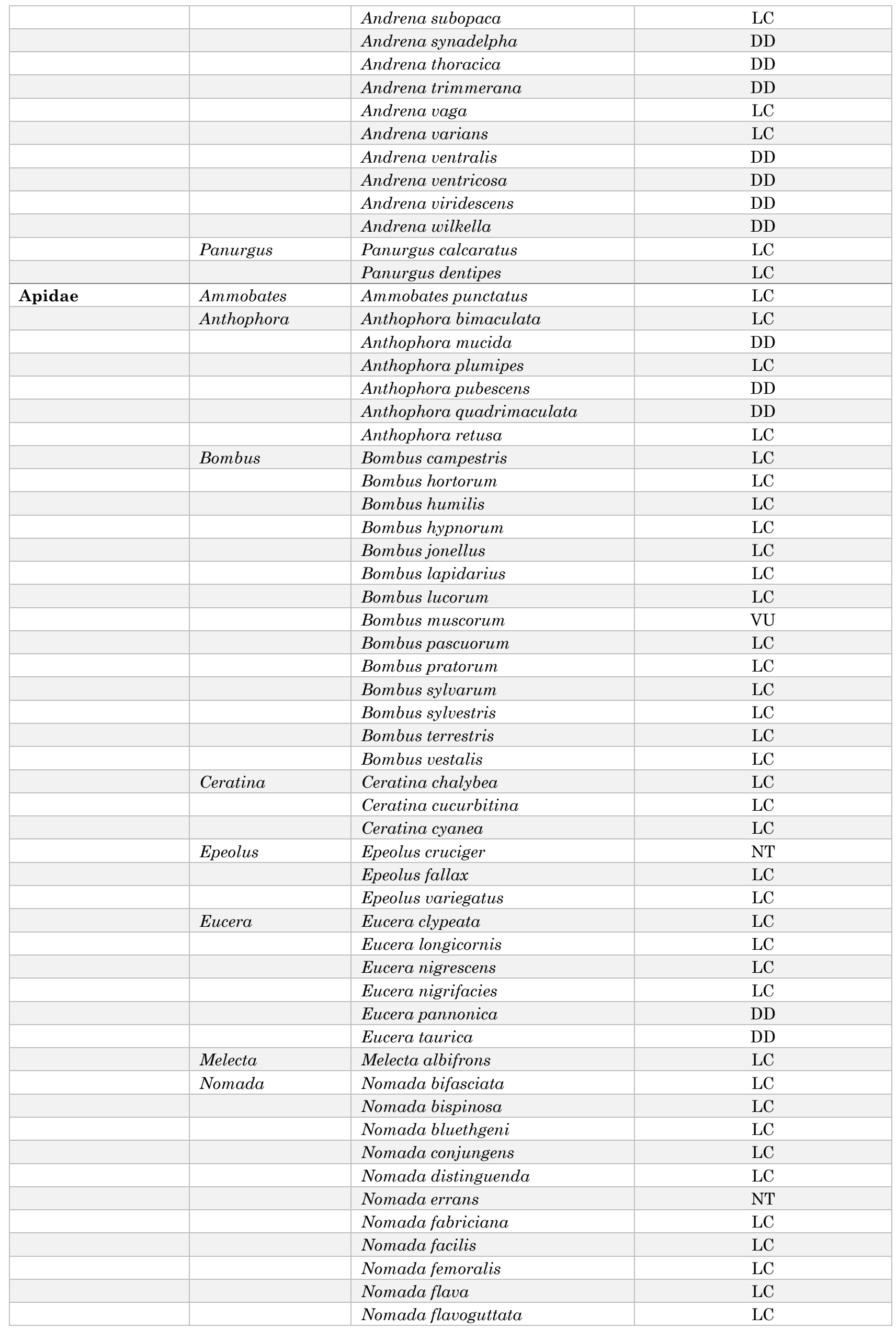




\begin{tabular}{|c|c|c|c|}
\hline & & Nomada flavopicta & $\mathrm{LC}$ \\
\hline & & Nomada fucata & $\mathrm{LC}$ \\
\hline & & Nomada fulvicornis & $\mathrm{LC}$ \\
\hline & & Nomada furva & DD \\
\hline & & Nomada goodeniana & $\mathrm{LC}$ \\
\hline & & Nomada guttulata & $\mathrm{LC}$ \\
\hline & & Nomada hirtipes & $\mathrm{LC}$ \\
\hline & & Nomada kohli & $\mathrm{LC}$ \\
\hline & & Nomada lathburiana & $\mathrm{LC}$ \\
\hline & & Nomada leucophtalma & $\mathrm{LC}$ \\
\hline & & Nomada lisenmaieri & $\mathrm{LC}$ \\
\hline & & Nomada melathoracica & $\mathrm{LC}$ \\
\hline & & Nomada panurgina & $\mathrm{LC}$ \\
\hline & & Nomada panzeri & $\mathrm{LC}$ \\
\hline & & Nomada rubiginosa & $\mathrm{LC}$ \\
\hline & & Nomada ruficornis & $\mathrm{LC}$ \\
\hline & & Nomada rufipes & $\mathrm{LC}$ \\
\hline & & Nomada sexfasciata & $\mathrm{LC}$ \\
\hline & & Nomada sheppardana & $\mathrm{LC}$ \\
\hline & & Nomada signata & $\mathrm{LC}$ \\
\hline & & Nomada stigma & $\mathrm{LC}$ \\
\hline & & Nomada succincta & $\mathrm{LC}$ \\
\hline & & Nomada villosa & NT \\
\hline & & Nomada zonata & $\mathrm{LC}$ \\
\hline & Tetralonia & Tetralonia malvae & $\mathrm{LC}$ \\
\hline & Tetraloniella & Tetraloniella alticincta & $\mathrm{LC}$ \\
\hline & Xylocopa & Xylocopa valga & $\mathrm{LC}$ \\
\hline & & Xylocopa violacea & $\mathrm{LC}$ \\
\hline \multirow[t]{26}{*}{ Colletidae } & Colletes & Colletes cunicularius & $\mathrm{LC}$ \\
\hline & & Colletes daviesanus & $\mathrm{LC}$ \\
\hline & & Colletes fodiens & VU \\
\hline & & Colletes gallicus & $\mathrm{LC}$ \\
\hline & & Colletes hederae & $\mathrm{LC}$ \\
\hline & & Colletes hylaeiformis & $\mathrm{LC}$ \\
\hline & & Colletes similis & $\mathrm{LC}$ \\
\hline & Hylaeus & Hylaeus annularis & DD \\
\hline & & Hylaeus brevicornis & $\mathrm{LC}$ \\
\hline & & Hylaeus clypearis & $\mathrm{LC}$ \\
\hline & & Hylaeus communis & $\mathrm{LC}$ \\
\hline & & Hylaeus confusus & $\mathrm{LC}$ \\
\hline & & Hylaeus dilatatus & $\mathrm{LC}$ \\
\hline & & Hylaeus duckei & DD \\
\hline & & Hylaeus euryscapus & DD \\
\hline & & Hylaeus gibbus & $\mathrm{LC}$ \\
\hline & & Hylaeus gredleri & $\mathrm{LC}$ \\
\hline & & Hylaeus hyalinatus & $\mathrm{LC}$ \\
\hline & & Hylaeus incongruus & DD \\
\hline & & Hylaeus leptocephalus & $\mathrm{LC}$ \\
\hline & & Hylaeus nigritus & $\mathrm{LC}$ \\
\hline & & Hylaeus pictipes & $\mathrm{LC}$ \\
\hline & & Hylaeus punctatus & $\mathrm{LC}$ \\
\hline & & Hylaeus punctulatissimus & DD \\
\hline & & Hylaeus signatus & $\mathrm{LC}$ \\
\hline & & Hylaeus variegatus & $\mathrm{LC}$ \\
\hline \multirow[t]{3}{*}{ Halictidae } & Halictus & Halictus compressus & $\mathrm{LC}$ \\
\hline & & Halictus gr. simplex & \\
\hline & & Halictus langobardicus & $\mathrm{LC}$ \\
\hline
\end{tabular}




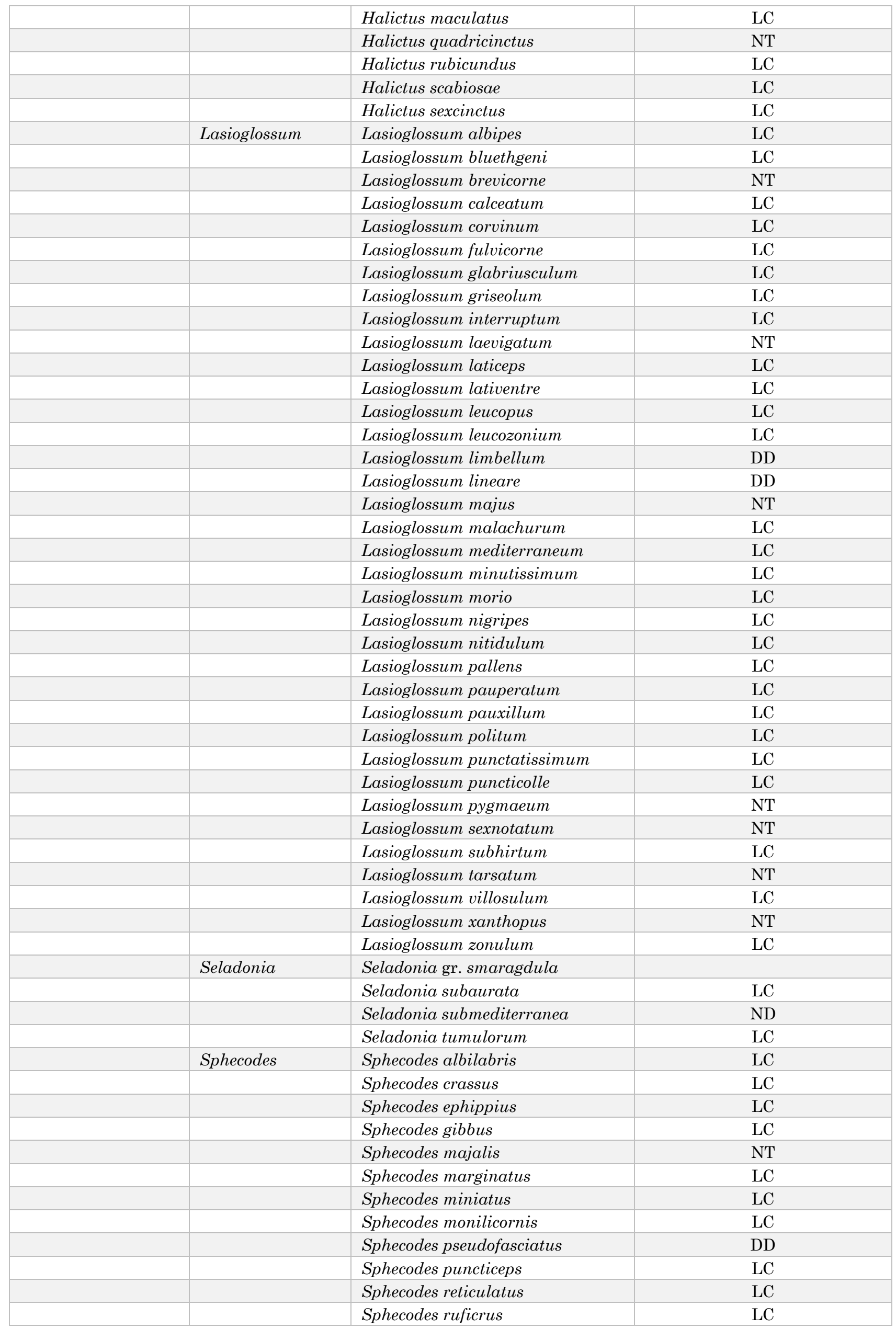




\begin{tabular}{|c|c|c|c|}
\hline & & Sphecodes rufiventris & $\mathrm{LC}$ \\
\hline & & Sphecodes spinulosus & NT \\
\hline & Vestitohalictus & Vestitohalictus pollinosus & $\mathrm{LC}$ \\
\hline \multirow[t]{49}{*}{ Megachilidae } & Aglaoapis & Aglaoapis tridentata & $\mathrm{LC}$ \\
\hline & Anthidiellum & Anthidiellum strigatum & $\mathrm{LC}$ \\
\hline & Anthidium & Anthidium manicatum & $\mathrm{LC}$ \\
\hline & & Anthidium oblongatum & $\mathrm{LC}$ \\
\hline & & Anthidium punctatum & $\mathrm{LC}$ \\
\hline & & Anthidium septemspinosum & DD \\
\hline & Chelostoma & Chelostoma distinctum & $\mathrm{LC}$ \\
\hline & & Chelostoma florisomne & $\mathrm{LC}$ \\
\hline & & Chelostoma rapunculi & $\mathrm{LC}$ \\
\hline & Coelioxys & Coelioxys afra & $\mathrm{LC}$ \\
\hline & & Coelioxys aurolimbata & $\mathrm{LC}$ \\
\hline & & Coelioxys brevis & $\mathrm{LC}$ \\
\hline & & Coelioxys conoidea & $\mathrm{LC}$ \\
\hline & & Coelioxys echinata & $\mathrm{LC}$ \\
\hline & & Coelioxys inermis & $\mathrm{LC}$ \\
\hline & & Coelioxys obtusa & $\mathrm{LC}$ \\
\hline & Heriades & Heriades crenulata & $\mathrm{LC}$ \\
\hline & & Heriades truncorum & $\mathrm{LC}$ \\
\hline & Hoplitis & Hoplitis adunca & $\mathrm{LC}$ \\
\hline & & Hoplitis leucomelana & $\mathrm{LC}$ \\
\hline & & Hoplitis tridentata & $\mathrm{LC}$ \\
\hline & Lithurgus & Lithurgus chrysurus & $\mathrm{LC}$ \\
\hline & & Lithurgus cornutus & $\mathrm{LC}$ \\
\hline & Megachile & Megachile centuncularis & $\mathrm{LC}$ \\
\hline & & Megachile circumcincta & $\mathrm{LC}$ \\
\hline & & Megachile deceptoria & DD \\
\hline & & Megachile ericetorum & $\mathrm{LC}$ \\
\hline & & Megachile lagopoda & $\mathrm{LC}$ \\
\hline & & Megachile leachella & $\mathrm{LC}$ \\
\hline & & Megachile maritima & DD \\
\hline & & Megachile pilidens & $\mathrm{LC}$ \\
\hline & & Megachile rotundata & DD \\
\hline & & Megachile willughbiella & $\mathrm{LC}$ \\
\hline & Osmia & Osmia aurulenta & $\mathrm{LC}$ \\
\hline & & Osmia bicornis & $\mathrm{LC}$ \\
\hline & & Osmia brevicornis & $\mathrm{LC}$ \\
\hline & & Osmia caerulescens & $\mathrm{LC}$ \\
\hline & & Osmia cornuta & $\mathrm{LC}$ \\
\hline & & Osmia emarginata & $\mathrm{LC}$ \\
\hline & & Osmia gallarum & $\mathrm{LC}$ \\
\hline & & Osmia leaiana & $\mathrm{LC}$ \\
\hline & & Osmia niveata & $\mathrm{LC}$ \\
\hline & & Osmia submicans & $\mathrm{LC}$ \\
\hline & & Osmia versicolor & $\mathrm{LC}$ \\
\hline & & Osmia viridana & $\mathrm{LC}$ \\
\hline & Pseudoanthidium & Pseudoanthidium gr. nanum & \\
\hline & Stelis & Stelis breviuscula & $\mathrm{LC}$ \\
\hline & & Stelis phaeoptera & DD \\
\hline & & Stelis punctulatissima & $\mathrm{LC}$ \\
\hline \multirow{5}{*}{ Melittidae } & Dasypoda & Dasypoda hirtipes & $\mathrm{LC}$ \\
\hline & Macropis & Macropis europaea & $\mathrm{LC}$ \\
\hline & Melitta & Melitta leporina & $\mathrm{LC}$ \\
\hline & & Melitta nigricans & $\mathrm{LC}$ \\
\hline & & Melitta tricincta & NT \\
\hline
\end{tabular}


Ceci est d'autant plus vrai que le collecteur possède une expertise sur les abeilles, les résultats tendant alors à être comparables à ceux de collectes par coupelles (O'CONNOR et al. 2019). Les collectes au filet présentent de plus l'avantage d'apporter des informations sur le comportement des abeilles collectées et d'approfondir les connaissances sur leur biologie (POPIC et al. 2013). Aussi, la plante butinée et les relations hôte-parasite ont été notées le cas échéant.

Les spécimens ont été préparés, identifiés au niveau du genre puis envoyés à des spécialistes pour identification à l'espèce. Le référentiel taxonomique utilisé est TaxRef version 12 (GARGOMINY et al. 2018). Les informations sur la biologie et la distribution des espèces sont issues du site Internet Atlas Hymenoptera, de références variées (AMIET et al. 1999, 2001, 2004, 2007, 2010 ; FALK \& LEWINGTON 2015; ElSE \& EDWARDS 2018; MiCHEZ et al. 2019; Observatoire des Abeilles 2018) et de dires d'experts (communications personnelles de Matthieu AubERT, Eric Dufrêne et David GENOUD).

\section{Résultats et discussion}

Davantage que de décrire de façon exhaustive les données obtenues lors de cette étude, notre objectif ici est d'en donner quelques informations synthétiques et de présenter de façon plus détaillée les résultats marquants de quelques-uns des sites étudiés. La liste complète des espèces collectées est fournie dans le tableau II (pages 6670).

\section{$\underline{\text { Richesse spécifique }}$}

Au total, 12666 spécimens d'abeilles sauvages ont été collectés au cours de ces cinq années d'étude et 278 espèces ont été identifiées (tableau II) : 256 espèces pour 9776 spécimens en Loire-Atlantique, 119 espèces pour 1240 spécimens en Maine-etLoire et 130 espèces pour 1650 spécimens en Vendée. Les espèces appartiennent aux six familles d'abeilles présentes en France (Andrenidae, Apidae, Colletidae, Halictidae, Megachilidae et Melittidae) et représentent 35 genres différents.

À ce stade, la proportion des spécimens identifiés à l'espèce est de $64 \%$ pour la Loire-Atlantique et de $87 \%$ pour chacun des deux autres départements. Aussi, avec l'avancée des identifications, le nombre d'espèces va probablement augmenter, particulièrement en Loire-Atlantique.

Le nombre moyen d'espèces détectées par commune est de 45 et va de 11 pour La Ferrière (85) à 134 pour Nantes. Les efforts d'échantillonnage et les milieux étudiés étant très variables d'une commune à l'autre, il n'est pas possible de comparer entre elles les richesses spécifiques. Le tableau I (page 65) indique le nombre d'espèces détectées au sein de chaque commune grâce à cette étude. Selon l'intensité de l'effort d'échantillonnage, le niveau des connaissances peut être considéré comme plus ou moins bon ( $c f$. code couleur du tableau I page 65).

Si l'on considère les 23 communes avec un unique site échantillonné une seule année (avec cinq à huit sessions de collecte), on observe qu'en moyenne 33 espèces sont détectées, avec un minimum de 12 à Thiré (85) et un maximum de 62 à Gétigné (44).

\section{Espèces communes et espèces rares}

La figure 3 (page 72) présente les 15 espèces les plus répandues (abondance quantifiée en nombre de communes où elles ont été prélevées). Plusieurs espèces sociales des genres Bombus, Halictus, Lasioglossum et Seladonia (figure 4) figurent parmi les espèces les plus répandues. Certaines espèces solitaires se révèlent également très communes : Lasioglossum leucozonium, Andrena flavipes, Anthophora plumipes, Eucera nigrescens et Hylaeus communis (figure 5).

À l'opposé, de nombreuses espèces se retrouvent en faibles effectifs dans le jeu de données : pour 103 espèces, c'est seulement un à trois spécimens qui ont été collectés (un seul spécimen pour 53 espèces, deux spécimens pour 29 espèces, trois spécimens pour 21 espèces).

En termes de nombre de sites, 66 espèces n'ont été collectées que sur un seul des 84 sites, 102 espèces sur deux à cinq sites. Seules 11 espèces ont été collectées dans plus de la moitié des sites (soit 42 sites ou plus). 


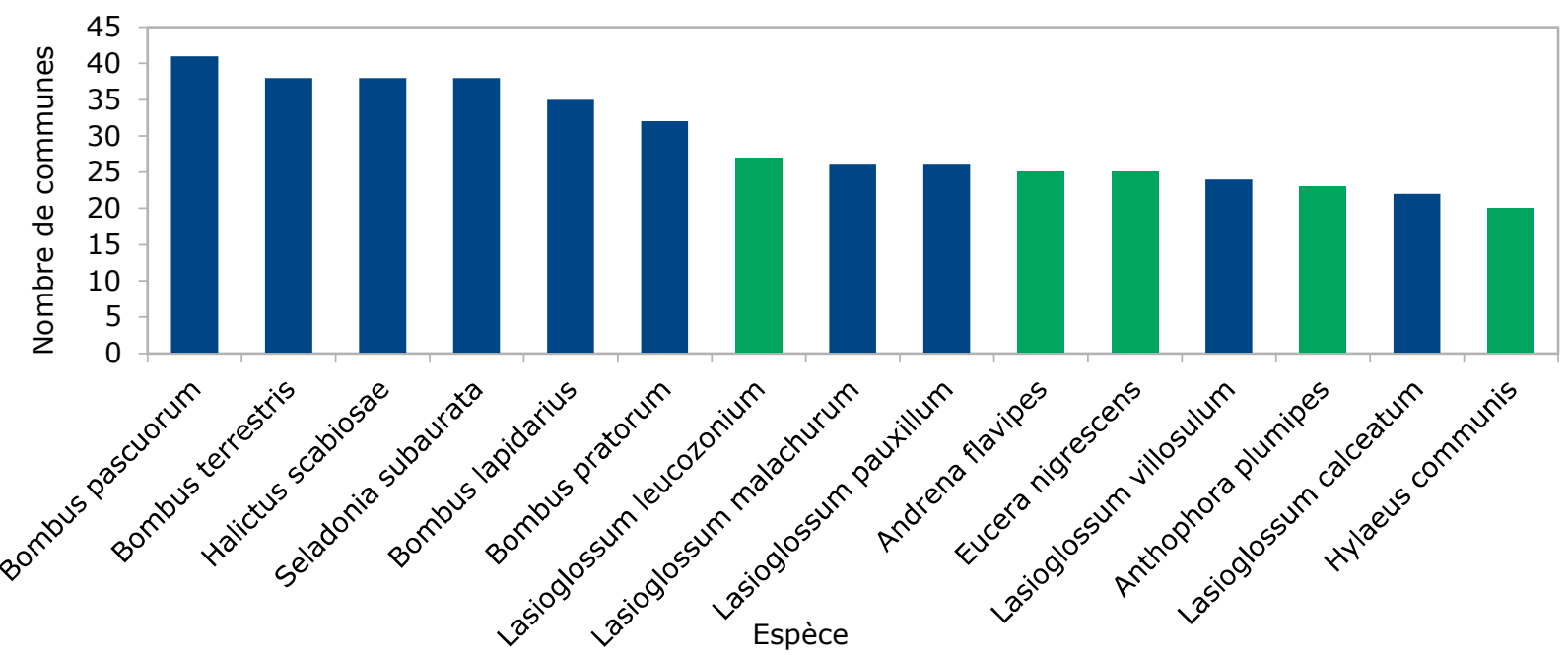

Figure 3. Les 15 espèces les plus répandues du jeu de données (en nombre de communes dans lesquelles l'espèce a été détectée). Les espèces sociales sont figurées en bleu, les solitaires en vert.

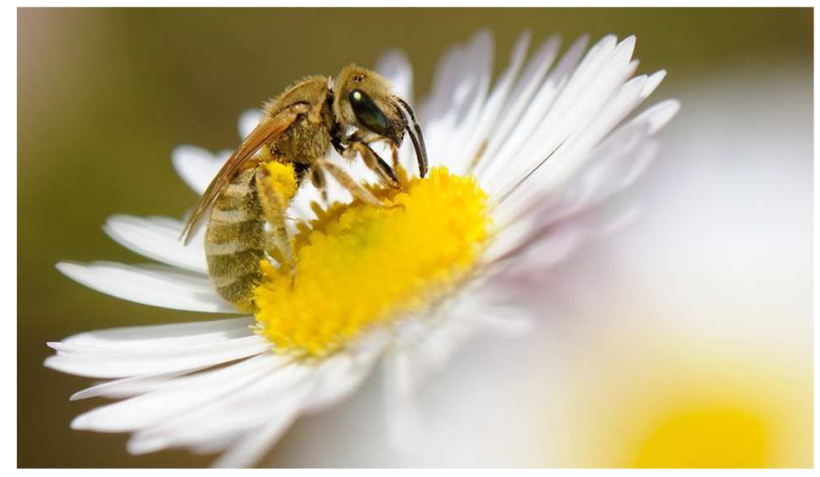

Figure 4. Seladonia subaurata, une des espèces les plus répandues dans cette étude (détectée dans 38 des 43 communes étudiées). Il s'agit d'une espèce sociale et polylectique de la famille des Halictidae. Photo Erwan BALANÇA.

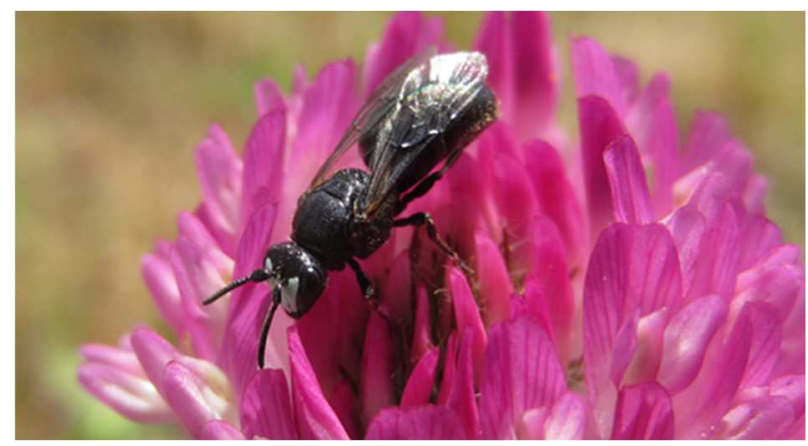

Figure 5. Un spécimen du genre Hylaeus (Colletidae). Dix-neuf espèces du genre Hylaeus ont été collectées lors de l'étude. Trois d'entre elles, Hylaeus clypearis, $H$. leptocephalus et $H$. punctatus, n'étaient jusqu'alors pas connues du Massif armoricain. Photo Olivier LAMBERT.

Plusieurs des espèces rares du jeu de données sont des espèces connues dans le sud de la France mais qui n'avaient pas été détectées jusqu'alors dans des localités aussi septentrionales. Il s'agit par exemple de Nomada bispinosa, N. bluethgeni, $N$. lisenmaieri et Andrena simontornyella (figure 6).

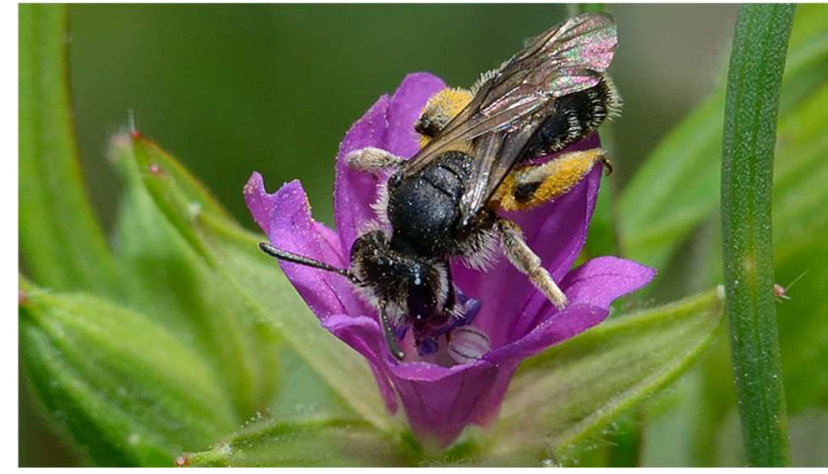

Figure 6. Andrena simontornyella (Andrenidae), un exemple d'espèce connue du sud de la France qui n'avait jusqu'alors pas été détectée dans des localités aussi septentrionales (probablement du fait de leur rareté et du manque de prospection). Photo David GENOUD.

Deux espèces sont classées vulnérables, donc menacées, dans la liste rouge des abeilles d'Europe (NIETO et al. 2014 ; tableau II pages 6670) : Bombus muscorum (figure 7 ; trois spécimens aux Moutiers-en-Retz [44]) et Colletes fodiens (deux spécimens aux Moutiers-en-Retz). Seize espèces sont classées quasi-menacées et 199 en préoccupation mineure. Faute de connaissances suffisantes, le statut des autres espèces n'est pas évalué dans la liste rouge.

Dans la liste rouge des bourdons de LoireAtlantique (MAHÉ 2015), Bombus jonellus (un spécimen à Angers) est classé vulnérable; $B$. humilis (un spécimen à Mauves-sur-Loire, un à Préfailles et trois à Pornic, communes toutes trois situées en Loire-Atlantique), B. muscorum (trois spécimens aux Moutiers-en-Retz) et B. sylvarum (un spécimen à Basse-Goulaine [44] et au Pellerin [44], trois à Angers) sont classés quasi-menacés. 


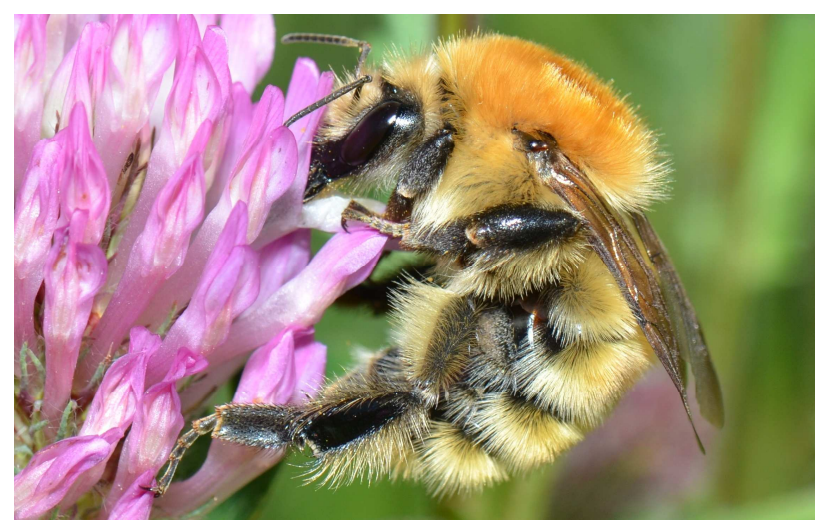

Figure 7. Bombus muscorum (Apidae), espèce classée vulnérable dans la liste rouge des abeilles d'Europe et présente aux Moutiers-en-Retz (dune du Collet et marais de Lyarne). Photo Gilles MAHÉ.

Un apport important pour la connaissance des abeilles du Massif armoricain

Notre étude apporte des connaissances sur la distribution géographique de 278 espèces d'abeilles en Pays de la Loire, contribuant ainsi à l'établissement des listes départementales des abeilles du Massif armoricain (Observatoire des Abeilles 2018) et permettant d'approfondir, pour certaines espèces, les connaissances à l'échelle nationale.

Elle a permis de révéler la présence dans le Massif armoricain (Bretagne, Pays de la Loire et ancienne Basse-Normandie) de 15 espèces qui n'y étaient jusqu'alors pas mentionnées, selon nos informations : Andrena florivaga, Chelostoma distinctum, Coelioxys obtusa, Eucera clypeata, E. pannonica, Hylaeus clypearis, $H$. leptocephalus, $H$. punctatus, Lasioglossum tarsatum, Nomada errans, $N$. furva, $N$. lisenmaieri, $N$. rubiginosa, $N$. villosa et Sphecodes pseudofasciatus.

La moitié de ces espèces ont été détectées sur des sites littoraux (Eucera clypeata à Pornic, E. pannonica, Nomada errans, $N$. rubiginosa aux Moutiers-en-Retz, Nomada furva et Chelostoma distinctum à Guérande), les autres parfois en plein cœur de ville (Hylaeus clypearis, H. leptocephalus et $H$. punctatus collectés au Jardin des Plantes de Nantes et Sphecodes pseudofasciatus au parc Balzac à Angers).

Diversité des milieux étudiés et caractéristiques des assemblages

Les sites littoraux dunaires des Moutiers-en-Retz (44)

La dune du Collet et le marais adjacent de Lyarne sont situés sur la façade littorale des Moutiers-en-
Retz, commune la plus au sud du littoral de LoireAtlantique. Le premier site a été échantillonné lors de trois années successives, de 2014 à 2016 (20 sessions de collecte au total), le second en 2014 et 2016 (13 sessions). Au total, 834 spécimens ont été collectés et 90 espèces identifiées. Les collectes sont dominées par Lasioglossum malachurum, Bombus terrestris et Andrena flavipes, trois espèces qui sont aussi parmi les plus abondantes du jeu de données global.

Outre la richesse spécifique élevée observée sur ces sites, nous avons constaté la présence de plusieurs espèces remarquables :

- Bombus muscorum : cette espèce est classée vulnérable dans la liste rouge européenne (NIETO et al. 2014) et quasi-menacée dans la liste rouge des bourdons de Loire-Atlantique (MAHÉ 2015). Sa préservation dans le Massif armoricain (où elle se trouve principalement dans les zones de marais) semble être un facteur-clé de sa préservation à l'échelle nationale, voire européenne. Lors de cette étude, elle n'a été trouvée qu'au marais de Lyarne (deux spécimens) et à la dune du Collet (un spécimen).

- Eucera taurica (figure 8) : dans cette étude, cette espèce très localisée dans le nord de la France n'a été trouvée qu'à la dune du Collet. Elle semble y être relativement abondante puisqu'elle y a été collectée lors des trois années, avec 17 spécimens au total. C'est une grosse eucère dont les femelles collectent le pollen préférentiellement sur les Astéracées (centaurées, cirses et scabieuses notamment).

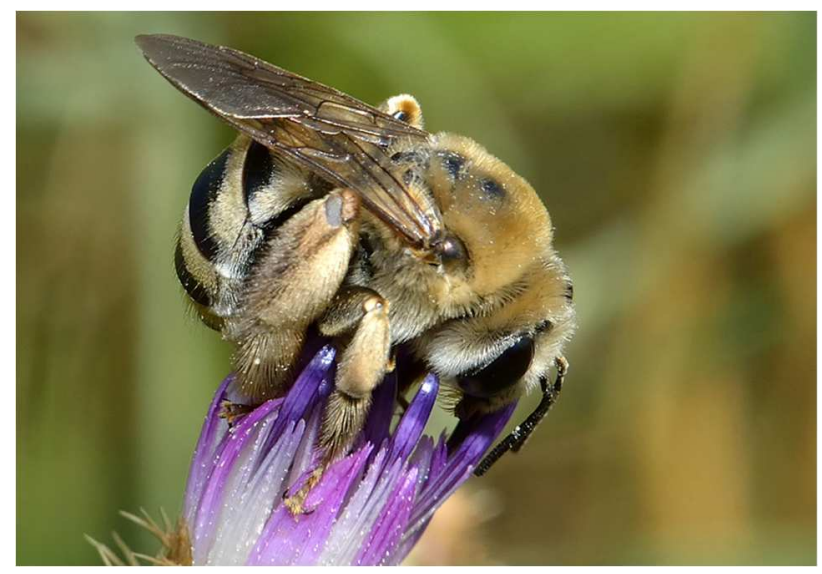

Figure 8. Eucera taurica (Apidae), espèce rare en France détectée à la dune du Collet (Les Moutiers-enRetz, 44) où elle semble relativement abondante. C'est une espèce oligolectique, butinant préférentiellement les Astéracées telles que cirses et centaurées. Photo David GENOUD. 
- Eucera pannonica : cette espèce, très localisée partout en France, n'a été détectée qu'à la dune du Collet lors de cette étude (deux spécimens).

- Nomada errans : un seul spécimen de cette espèce, classée quasi-menacée dans la liste rouge européenne, a été collecté lors de cette étude. Son hôte connu est Andrena nitidiuscula, espèce oligolectique sur Apiacées qui n'est connue que de la Loire-Atlantique dans le Massif armoricain et qui n'a été collectée que sur deux communes lors de cette étude, Les Moutiers-en-Retz et Guérande.

- Nomada rubiginosa : cette espèce est cantonnée à la façade atlantique et atteint là la limite nord de son aire de distribution connue (un seul spécimen collecté lors de cette étude).

- Megachile deceptoria : cette espèce que l'on pensait localisée au littoral méditerranéen a été récemment découverte sur le littoral atlantique. Dans le cadre de cette étude, elle a été collectée sur deux communes, Les Moutiersen-Retz et Guérande (site de Kersalio).

- Plusieurs andrènes peu communes ou spécialistes (oligolectiques) ont été détectées (Andrena flavilabris, A. labialis, A. livens, $A$. nitidiuscula, A. pandellei, $A$. ranunculi et $A$. schencki) ainsi qu'A. ovatula, classée quasimenacée dans la liste rouge européenne mais plutôt commune en France.

- En ce qui concerne les collètes, on retrouve le cortège littoral déjà étudié plus au nord du département sur la presqu'île guérandaise (MAHÉ 2009). Sont présentes les communes Colletes cunicularius, C. hederae et C. similis, ainsi que les moins communes $C$. fodiens (classée vulnérable dans la liste rouge européenne), C. gallicus et C. hylaeiformis. Colletes fodiens et $C$. gallicus ont été trouvées uniquement aux Moutiers-en-Retz dans cette étude.

- Lasioglossum brevicorne : il s'agit de l'une des peu nombreuses espèces oligolectiques dans le genre Lasioglossum (sur Astéracées pour cette espèce). Cette espèce localisée en France (sur sols aérés et détritiques meubles et alluvions en contexte thermophile) a également été notée dans cette étude sur les communes de Gétigné, Rezé et Vertou (44) et dans deux parcs d'Angers. Elle est classée quasi-menacée dans la liste rouge européenne.

- Lasioglossum corvinum : cette espèce est surtout présente en France sur le pourtour méditerranéen. Elle est localisée ailleurs en
France et semble absente au nord de la Seine. Dans cette étude, elle a été collectée uniquement sur le littoral (Les Moutiers-enRetz, Guérande et Pornic).

- Lasioglossum pauperatum et L. xanthopus (classé quasi-menacé dans la liste rouge européenne) font également partie des espèces remarquables de ces sites.

- Melitta leporina (figure 9) : un spécimen de cette espèce oligolectique sur Fabacées, avec des préférences pour les genres Medicago, Melilotus et Trifolium, a été collecté à la dune du Collet.

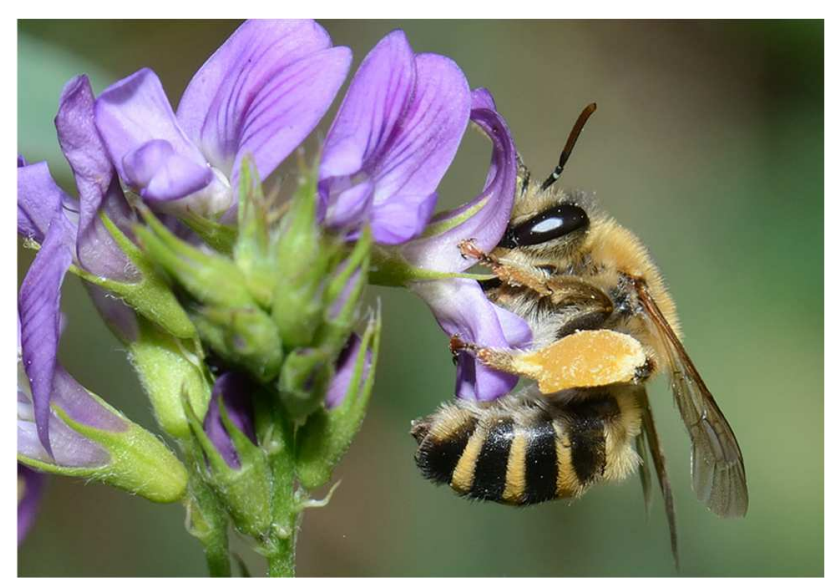

Figure 9. Melitta leporina (Melittidae), espèce oligolectique sur Fabacées, collectée dans plusieurs types d'habitats, y compris en plein cœur des villes (exemples : quartier Bottière-Chénaie et douves du Château des Ducs de Bretagne à Nantes). Photo David GeNoud.

Nantes : des pelouses et toits végétalisés du centreville aux prairies et bois de la périphérie

À Nantes, les collectes ont été réalisées dans des milieux variés : en plein centre-ville (pelouse bordant le Château des Ducs, massifs ornementaux sur la place de Bretagne ou sur la place Saint-Pierre près de la cathédrale), sur le toit végétalisé d'une école, dans des parcs urbains (Jardin des Plantes, parc des quartiers BottièreChénaie et Grand-Blottereau et espaces verts autour des bâtiments de la Direction Départementale des Territoires et de la Mer DDTM) et dans des zones moins anthropisées (bois des Anses, marais de Gachet, Prairie de Mauves et vallée de la Chézine). Douze sites ont été échantillonnés, tous durant une année, sauf les parcs de Bottière-Chénaie et du GrandBlottereau, échantillonnés pendant respectivement deux et trois ans. Au total, 1893 spécimens ont été collectés, représentant 134 espèces. 
En plein cœur du centre-ville (Château, places de Bretagne et Saint-Pierre), 30 espèces ont été collectées. Les plus abondantes ne sont pas les espèces les plus abondantes du jeu de données global (bourdons communs et Halictidae sociaux) mais Anthophora plumipes et Hylaeus hyalinatus. Ces deux espèces solitaires nidifient dans divers types de cavités, notamment les interstices dans les murs en pierres ou, pour $H$. hyalinatus, les tiges de végétaux ou les trous dans le bois mort. Plusieurs espèces rares ou spécialistes ont été notées : Anthophora pubescens (peu commune en France), Colletes daviesanus (oligolectique sur Astéracées), Megachile lagopoda (assez peu commune en France) ou Melitta leporina (oligolectique sur Fabacées).

Sur le toit végétalisé de l'école Aimé Césaire, sur l'île de Nantes, 12 espèces ont été notées (pour 47 spécimens au total). Cette école a été construite en 2012 et deux types de végétation ont été mis en place sur son toit : un milieu de type " dunaire" avec un sol sableux et une implantation dense de graminées (oyat), de laîche des sables ou encore de géranium sanguin et un milieu de type " lande et friche " avec un sol argileux localement enrichi de vraie terre de bruyère et une densité de semis moins importante que dans la zone "dunaire " pour permettre une colonisation naturelle par d'autres espèces végétales.

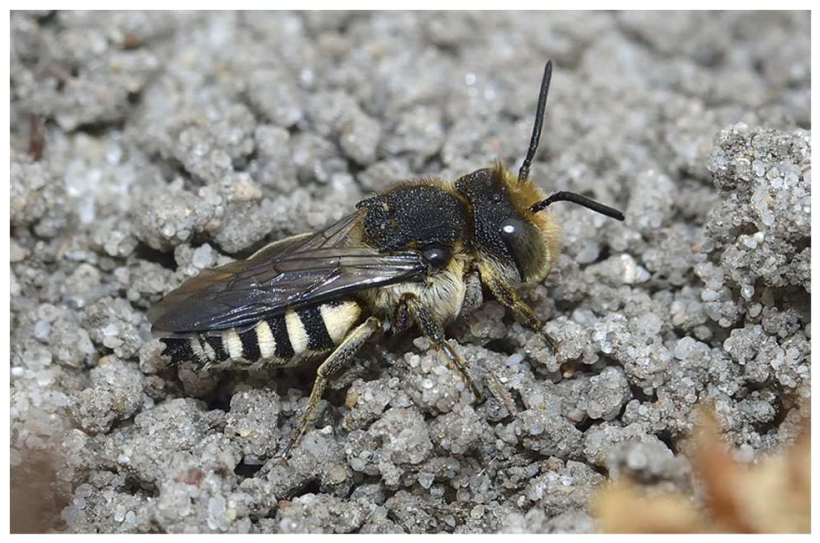

Figure 10. Coelioxys conoidea (Megachilidae), espèce cleptoparasite relativement abondante sur le toit végétalisé de l'école Aimé Césaire sur l'île de Nantes. Photo David GeNoud.

La diversité fonctionnelle des abeilles paraît intéressante, notamment au regard de l'âge du site (école construite en 2012 et collectes réalisées en 2015). Sont présentes à la fois des espèces terricoles et cavicoles. De plus, une espèce coucou (Coelioxys conoidea, figure 10, qui parasite les nids de plusieurs espèces du genre Megachile) y est relativement abondante. Les espèces rencontrées sont communes en France, sauf Lasioglossum leucopus (peu commun).

Dans les parcs (Jardin des Plantes, parc des quartiers Bottière-Chénaie et Grand-Blottereau et espaces verts autour des bâtiments de la DDTM), 1050 spécimens ont été collectés pour un total de 92 espèces. L'unique spécimen de l'étude de Melitta tricincta a été collecté dans le parc du Grand-Blottereau. Cette espèce classée quasimenacée dans la liste rouge européenne est oligolectique sur les odontites, notamment Odontites vernus. Dans le Massif armoricain, elle est connue uniquement de Loire-Atlantique et des trois départements de (l'ancienne) BasseNormandie. M. leporina est quant à elle présente dans les parcs de Bottière-Chénaie et du GrandBlottereau. Plusieurs andrènes peu communes ou spécialistes ont été détectées (Andrena cinerea, $A$. lagopus, A. rhenana, A. schencki et A. wilkella). Dans le Jardin des Plantes, situé à proximité du centre-ville, un spécimen d'Anthophora pubescens et un autre de Megachile lagopoda ont été collectés. C'est aussi au Jardin des Plantes qu'ont été collectés les uniques spécimens pour cette étude - constituant les premières mentions pour le Massif armoricain - d'Hylaeus leptocephalus et $H$. punctatus. Citons enfin la présence dans le parc de Bottière-Chénaie de Lasioglossum griseolum, une espèce méditerranéenne rare dans le nord de la France et la présence dans le parc du GrandBlottereau de Sphecodes majalis (classé quasimenacé dans la liste rouge européenne).

Dans les zones moins anthropisées (bois des Anses, marais de Gachet, Prairie de Mauves, vallée de la Chézine) ont été recensées 84 espèces pour 1254 spécimens. Parmi elles, notons la présence d'Andrena florivaga, A. vaga, $A$. ranunculi et Lasioglossum limbellum à la Prairie de Mauves, de Macropis europaea au marais de Gachet, d'Andrena labialis et Melitta leporina dans la vallée de la Chézine, de Lasioglossum laevigatum (classé quasi-menacé dans la liste rouge européenne) à la fois au marais de Gachet et dans la vallée de la Chézine et enfin d'Epeolus cruciger (classé quasi-menacé dans la liste rouge européenne), Lasioglossum griseolum et $L$. pauperatum au bois des Anses. Andrena florivaga, espèce très rare en France, était jusqu'alors inconnue de la moitié ouest de la France. Selon BÉNON \& PRAZ (2016), elle semble en expansion en Europe. 
La Roche-sur-Yon: entre centre urbain et périphérie agricole

À La Roche-sur-Yon, huit sites ont été étudiés, cinq localisés en pleine ville (place Napoléon, boulevard Gaston Deferre ou encore parc des Oudairies) et trois en zone agricole (Lycée Nature, vallée de l'Yon et ancien centre Beautour). Les collectes comptent 1318 spécimens, dont plus de la moitié (55\%) proviennent des trois sites localisés en zone agricole. Au total, 120 espèces ont été collectées parmi lesquelles les plus remarquables sont Andrena cinerea, A. ferox, A. fulvida (classée quasi-menacée dans la liste rouge européenne), $A$. lagopus, A. simontornyella, A. wilkella, Lasioglossum laevigatum (classé quasi-menacé dans la liste rouge européenne), L. pauperatum, $L$. xanthopus (classé quasi-menacé dans la liste rouge), Melitta leporina, Nomada bispinosa, $N$. signata et Sphecodes majalis. Andrena ferox, collectée uniquement dans le parc des Oudairies lors de cette étude, a la particularité de collecter le pollen sur les arbres, en particulier sur les chênes (Quercus spp.) et les érables (notamment l'érable sycomore Acer pseudoplatanus). Dans le Massif armoricain, cette espèce n'est connue que des Pays de la Loire (en Loire-Atlantique, Maine-et-Loire et Vendée) (Observatoire des Abeilles 2018).

Angers : un effort d'échantillonnage moindre mais plusieurs découvertes

À Angers, troisième ville de taille importante étudiée, les collectes ont été moins nombreuses qu'à Nantes et La Roche-sur-Yon (727 spécimens au total). Les collectes y ont été réalisées dans cinq sites : trois parcs (parcs de Bellefontaine, du Pin et Balzac), les jardins d'un ancien couvent (" prairie Jeanne Jugan ») et les espaces verts d'un lotissement du quartier Molière. Au total, 91 espèces ont été détectées parmi lesquelles les plus remarquables sont :

- Bombus jonellus : cette espèce est rare en France et classée vulnérable dans la liste rouge des bourdons de Loire-Atlantique, où les landes à Éricacées sont son habitat de prédilection. Un seul spécimen a été collecté au cours de cette étude, dans le parc du Pin à Angers.

- Bombus sylvarum : trois spécimens de cette espèce en raréfaction dans plusieurs pays européens (MicHEZ et al. 2019) et classée quasimenacée dans la liste rouge des bourdons de
Loire-Atlantique ont été collectés dans la prairie Jeanne Jugan.

- Plusieurs andrènes rares ou spécialisées ont été collectées telles qu'Andrena apicata, A. rhenana ou A. simontornyella.

- Dans le parc Balzac ont été collectés les uniques exemplaires pour le jeu de données des espèces suivantes: Sphecodes pseudofasciatus (il s'agit de la première mention de l'espèce dans le Massif armoricain) et Xylocopa valga (espèce connue actuellement de trois départements seulement dans le Massif armoricain : la Loire-Atlantique, le Maine-etLoire et la Mayenne) et Vestitohalictus pollinosus. Cette espèce thermophile du pourtour méditerranéen et $\mathrm{du}$ littoral atlantique est présente au moins jusqu'aux îles morbihannaises mais semble absente d'un grand tiers nord de la France.

- Enfin, notons également la présence d'Anthophora pubescens et Megachile lagopoda (dans le parc Balzac), Nomada bispinosa (dans le quartier Molière), ainsi que de quatre espèces classées quasi-menacées dans la liste rouge européenne : Sphecodes majalis (dans le parc Balzac), Lasioglossum brevicorne (dans les parcs Balzac et de Bellefontaine), $L$. laevigatum (dans le parc Balzac et le quartier Molière) et $L$. pygmaeum (dans les trois parcs étudiés).

Le golf de Saint-Sébastien-sur-Loire (44): un cortège relativement riche d'andrènes

Ce golf a été créé en 2007 sur une île de la Loire située à Saint-Sébastien-sur-Loire, commune limitrophe de Nantes. S'il n'existe pas d'inventaire des abeilles précédant sa création, nos données indiquent qu'aujourd'hui le site héberge une faune d'abeilles relativement riche. Lors des collectes réalisées en 2016, 55 espèces y ont été recensées, ce qui n'est pas négligeable étant donné le petit nombre de spécimens collectés (164). Parmi elles, on compte des espèces en limite nord d'aire de répartition (Andrena simontornyella), des espèces spécialistes ou peu communes (Andrena lagopus, A. ranunculi, $A$. wilkella, A. vaga, Melitta nigricans, Lasioglossum pauperatum, Nomada conjunguens, N. stigma ou Sphecodes spinulosus, cette dernière espèce étant classée quasi-menacée dans la liste rouge européenne). 
Un intérêt particulier du site réside dans la présence d'une bourgade d'Andrena vaga (figure 11) composée de plusieurs dizaines de nids. Cette espèce oligolectique sur saules (en particulier Salix caprea selon MicHEz et al. 2019) est en effet très localisée dans l'ouest de la France. Lors de cette étude, elle a également été détectée sur le site de la Prairie de Mauves (Nantes), sur l'autre rive de la Loire, en face du golf. À la suite de cet inventaire, les agents d'entretien du golf ont pris en compte la présence des abeilles dans la gestion de certains espaces du site (décalage de dates d'intervention, maintien de zones ouvertes ou encore préservation de ressources alimentaires).

Parmi les espèces remarquables recensées sur des sites de bords de Loire, citons également Andrena argentata, espèce bivoltine dont le seul spécimen du jeu de données a été collecté à Trentemoult (Rezé, 44).

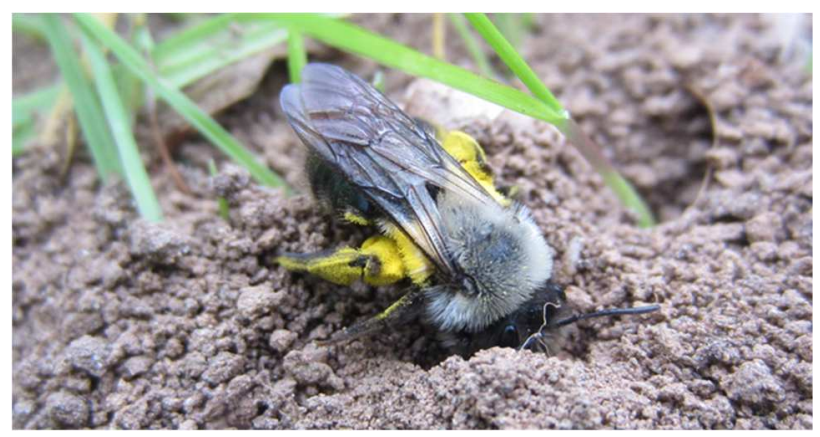

Figure 11. Andrena vaga (Andrenidae), espèce oligolectique sur saules. Ici une femelle à l'entrée de son nid, au golf de Saint-Sébastien-sur-Loire. Photo Olivier LAMBERT.

Les zones boisées : la prédominance des espèces printanières

Trois sites boisés ont été échantillonnés, tous en Loire-Atlantique : le bois de Touffou à Vertou, Les Coulées au Cellier et le site des Rocs de Gascaigne à Guémené-Penfao. Les collectes ont mis en évidence la présence de 58 espèces pour 315 spécimens et sont dominées par les genres Bombus (33\% des spécimens), Andrena (22\%) et Nomada (19\%). Ces trois genres représentent au total près des trois quarts des spécimens collectés. Sur l'ensemble du jeu de données, ces proportions sont respectivement de 19,17 et $4 \%$, soit $40 \%$ pour les trois genres. Ces sites boisés sont donc dominés par les espèces printanières (andrènes, nomades) et les espèces présentant une affinité plus importante que les autres pour les zones boisées (bourdons).
Parmi les autres groupes, citons la collecte de cinq espèces classées quasi-menacées dans la liste rouge européenne : Epeolus cruciger aux Rocs de Gascaigne, Sphecodes majalis au Cellier, Lasioglossum pygmaeum au bois de Touffou, $L$. majus, espèce peu fréquente en France détectée au bois de Touffou et aux Rocs de Gascaigne et $L$. tarsatum, dont l'unique spécimen de l'étude a été collecté aux Rocs de Gascaigne. Cette espèce du nord de l'Europe, rare en France, est connue uniquement de la Loire-Atlantique dans le Massif armoricain.

En considérant les trois sites boisés dans leur ensemble, on observe que la Ronce (Rubus sp.) est l'espèce végétale la plus butinée, suivie par les asphodèles (Asphodelus sp.).

Les relations flore-abeilles

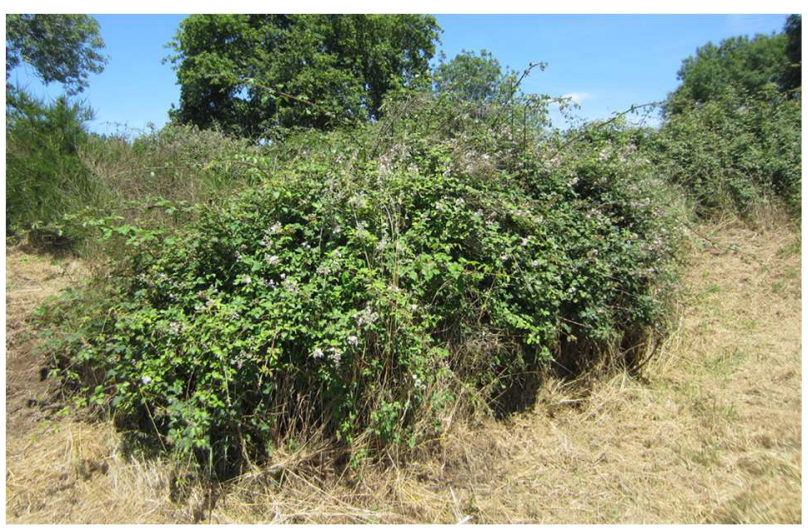

Figure 12. La ronce, espèce végétale sur laquelle a été capturé le plus grand nombre d'espèces d'abeilles $(N=75)$ lors de cette étude. Photo Olivier LAMBERT.

La grande majorité des spécimens collectés (85\%) l'ont été alors qu'ils visitaient une fleur. Les autres ont été capturés en vol ou posés, parfois près de leur site de nidification. Les spécimens ont été collectés sur plus de 400 espèces végétales différentes, sauvages ou ornementales. Les dix espèces comptant le plus de spécimens collectés sont la Porcelle enracinée Hypochaeris radicata (5,3 \% des spécimens), le Lotier commun Lotus corniculatus (4,1\%), le Pissenlit Taraxacum sp. $(4,0 \%)$, le Trèfle commun Trifolium pratense (3,3\%), la Ronce Rubus gr. fruticosus (3,1\%), la Pâquerette Bellis perennis (3,0 \%), les centaurées du groupe nigra, Centaurea gr. nigra (2,9\%), le Trèfle blanc Trifolium repens (2,9\%), le Liseron des champs Convolvulus arvensis (1,8\%) et la Renoncule âcre Ranunculus acris (1,4\%). Lorsque l'on établit le classement des plantes en fonction $\mathrm{du}$ nombre d'espèces d'abeilles collectées, on 
retrouve quasiment le même " top 10 " mais dans un ordre différent : la Ronce (75 espèces, figure 12), le Pissenlit (69 espèces), la Pâquerette (64 espèces), les Centaurées du groupe nigra (60 espèces), la Porcelle enracinée (55 espèces), le Lotier commun (54 espèces), le Trèfle commun (46 espèces), le Cirse des champs Cirsium arvense (43 espèces), la Renoncule âcre (43 espèces) et le Trèfle blanc (43 espèces).

Parmi cette liste de plantes, certaines sont très printanières (Pâquerette, Renoncule âcre, Pissenlit), d'autres un peu plus tardives (trèfles) ou de fin de printemps et d'été (Lotier commun, Ronce, Porcelle enracinée, Liseron des champs ou Cirse des champs).

Certaines plantes sont exploitées davantage par des abeilles généralistes (polylectiques), d'autres davantage par des spécialistes (oligolectiques). Ainsi, les Fabacées sont peu ou pas exploitées par des généralistes hormis des Apidae (Apis mellifera et Bombus spp.). C'est également vrai pour les renoncules, qui possèdent un large cortège de spécialistes (dont Andrena florivaga) - mais restent toutefois visitées pour la collecte du nectar par de nombreux Lasioglossum. À l'opposé, la précoce Pâquerette est généralement peu exploitée pour son pollen mais constitue une ressource en nectar importante. De la même façon, à cette latitude, la majorité des plantes estivales sont particulièrement recherchées par les abeilles pour leur disponibilité en nectar à une période où la disponibilité en ressource est critique. Aussi, parmi les abeilles collectées dans cette étude, il n'existe pas d'espèce spécialisée sur la ronce alors qu'elle demeure très attractive (pour les Lasioglossum, Hylaeus, Bombus ou les andrènes de deuxième génération), en plus de fournir des sites de nidification à certaines espèces des genres Hylaeus et Ceratina (FALK \& LEWINGTON 2015). Quant à elles, les Astéracées estivales (ici Cirse des champs, Porcelle enracinée ou centaurées) sont largement visitées pour leur nectar mais aussi pour leur pollen par des abeilles spécialistes estivales (Colletes, Megachilidae et espèces estivales d'Andrenidae notamment).

L'importance des suivis de long terme pour une bonne évaluation de la composition des communautés

La dune du Collet fait partie des sites qui ont été bien prospectés et ont révélé une communauté d'abeilles riche. Nous prenons ici ce site en exemple pour illustrer l'importance des suivis de long terme pour une bonne évaluation de la composition des communautés. Ce site a été prospecté en 2014, 2015 et 2016 , avec tous les mois entre mars et septembre une session de deux heures, par deux personnes en général. Au total, 427 spécimens ont été collectés et 61 espèces identifiées. Parmi elles, seules 13 ont été collectées lors des trois années d'étude (soit un cinquième des espèces environ), 13 l'ont été au cours de deux années et plus de la moitié $(\mathrm{n}=35)$ l'ont été au cours d'une seule année. La richesse estimée pour ce site est de 82,1 espèces selon l'estimateur Chao1 et 86,8 espèces selon l'estimateur ACE. L'effort d'échantillonnage employé, relativement conséquent, a donc permis de détecter respectivement environ 74 et $70 \%$ des espèces prédites pour ce site.

\section{Conclusion}

Cette étude a permis d'approfondir les connaissances sur la distribution géographique de 278 espèces en région Pays de la Loire. Au-delà de cet approfondissement des connaissances au niveau régional, de précieuses informations ont aussi été apportées sur la distribution de plusieurs espèces rares en France, dont la présence n'était jusqu'alors pas connue dans le Massif armoricain. Il s'agit notamment de plusieurs espèces d'affinité méditerranéenne qui atteignent ici leur limite nord de répartition, comme c'est le cas aussi pour d'autres espèces d'invertébrés (HERBRECHT et al. 2015). Les inventaires menés par le CVFSE continuent avec l'objectif de poursuivre les prospections sur certains sites (les îles de Loire par exemple) et de compléter le maillage de prospection sur la région. Le lecteur pourra également se référer au récent travail d'HERBRECHT et al. (2019) qui fournit de nouveaux éléments sur les abeilles présentes dans les différents départements des Pays de la Loire.

Nous avons montré l'intérêt majeur de certains sites, notamment les sites littoraux dunaires, pour la préservation des abeilles à l'échelle régionale. $\mathrm{Si}$ ce type de milieux naturels présente les conditions favorables à la présence de riches communautés, plusieurs sites urbains ou péri-urbains hébergent également des communautés relativement riches, avec certaines espèces spécialisées dans leurs besoins écologiques (sur cette thématique, voir aussi FORTEL et al. 2014 pour Lyon et son 
agglomération et ROPARS et al. 2018 pour Paris). Ces milieux ne doivent donc pas être négligés et les méthodes de gestion favorables aux abeilles doivent y être encouragées pour permettre aux populations de se maintenir.

\section{Remerciements}

Nous remercions vivement les spécialistes qui ont participé à l'identification des spécimens : Matthieu AUBERT, Éric DUFRÊNE, Gilles MAHÉ et Alain PAULY. Merci aux personnes qui ont participé aux collectes sur le terrain et au travail de laboratoire : Pascal Bonnin, Rémy CHIFFLET, Sébastien FARAU, Éléonore HAULOT, Marc HERVÉ, Marie LE BRAZIDEC, Axelle LE ChÊne, Morgan MuYlaERT, Nicolas NALPOWIK, Émeline TATA, Élodie ToutAIN, Nicolas VAN WyMEERSCH. Merci à Floriane FlACHER, Benoît GESLIN, Franck HERBRECHT, Tanguy JEAN et Gilles MAHÉ pour leur relecture du texte, et à Mehdi IssERTES pour la mise en page. Merci aux propriétaires et gestionnaires de sites pour en avoir permis et facilité l'accès. Les programmes dont cet article fait la synthèse ont été financés par la Région Pays de la Loire, le Conseil Départemental de LoireAtlantique, Nantes Métropole et la Ville de Nantes.

\section{Bibliographie}

Amiet F, Müller A \& Neumeyer R, 1999. Apidae 2 : Colletes, Dufourea, Hylaeus, Nomia, Nomioides, Rhophitoides, Rophites, Sphecodes, Systropha. Fauna Helvetica. Centre Suisse de Cartographie de la Faune. Neuchâtel, Suisse. $219 \mathrm{pp}$.

Amiet F, Herrmann M, MÜller A \& NeUMEYER R, 2001. Apidae 3 : Halictus, Lasioglossum. Fauna Helvetica. Centre Suisse de Cartographie de la Faune. Neuchâtel, Suisse. 208 pp.

Amiet F, HERRMANN M, MÜller A \& NEUMEYER R, 2004. Apidae 4 : Anthidium, Chelostoma, Coelioxys, Dioxys, Heriades, Lithurgus, Megachile, Osmia, Stelis. Fauna Helvetica. Centre Suisse de Cartographie de la Faune. Fauna Helvetica. Centre Suisse de Cartographie de la Faune. Neuchâtel, Suisse. $273 \mathrm{pp}$.
Amiet F, HerrmanN M, MÜller A \& NEUMEYER R, 2007. Apidae 5: Ammobates, Ammobatoides, Anthophora, Biastes, Ceratina, Dasypoda, Epeoloides, Epeolus, Eucera, Macropis, Melecta, Melitta, Nomada, Pasites, Tetralonia, Thyreus, Xylocopa. Fauna Helvetica. Centre Suisse de Cartographie de la Faune. Neuchâtel, Suisse. 356 pp.

Amiet F, Herrmann M, Müller A \& NeUmeYer R, 2010. Apidae 6 : Andrena, Melitturga, Panurginus, Panurgus. Fauna Helvetica. Centre Suisse de Cartographie de la Faune. Neuchâtel, Suisse. 316 pp.

BÉnON D \& Praz C, 2016. Deux nouvelles espèces d'abeilles sauvages pour l'entomofaune suisse : observation d'Andrena florivaga Eversmann, 1852 et Andrena fulvicornis Schenck, 1853 dans les pâturages jurassiens. Entomo Helvetica 9 : 69-78.

https://sciencesnaturelles.ch/uuid/d3590825-56e95881-b1e5a6b5b952be17? $\mathrm{r}=20170706115333 \_150$ 2366030_3d1c6c76-9cf2-5c23-abee-e47475b665c3 (consultation octobre 2020)

ELSE GR \& EDWARDS M, 2018. Handbook of the Bees of the British Isles (2-Volume Set). Ray Society Monographs. The Ray Society, Londres. 775 pp.

FALK S \& LEWINGTON R, 2015. Field guide of the bees of Great Britain and Ireland. British Wildlife Field Guides. Bloomsbury, Londres, 432 pp.

Fortel L, Henry M, Guilbaud L, Guirao AL, KuHLMANN M, Mouret H, Rollin O \& VAISSIÈRE BE, 2014. Decreasing abundance, increasing diversity and changing structure of the wild bee community (Hymenoptera: Anthophila) along an urbanization gradient. PLoS ONE 9(8): e104679.

https://doi.org/10.1371/journal.pone.0104679

Gargominy O, Tercerie S, RÉgnier C, RAMAGE T, DUPONT P, VANDEL E, DASZKIEWICZ P, Léotard G, Courtecuisse R, Canard A, LÉVÊque A, LEblOND S, DE MASSARY J-C, JOURDAN H, DEWYNTER M, HORELlOU A, NOËL P, Noblecourt T, Comolet J, Touroult J, yy BARBut J, RoMe Q, DELFosse E, BERNARD JF, BOCK B, MALÉCOT V, BOULlET V, HugONNOT 
V, ROBBERT GRADSTEIN S, LAVOCAT BERNARD E, Ah-Peng C, Moreau PA \& Lebouvier M, 2018. TAXREF v12.0, référentiel taxonomique pour la France. Muséum national d'Histoire naturelle, Paris.

https://inpn.mnhn.fr/telechargement/referentielE spece/taxref/12.0/menu (consultation octobre 2020)

HeRbreChT F, 2012. Suivi des hyménoptères fouisseurs de la zone écologique du terminal multivrac de Montoir-de-Bretagne (FR-44). Année 2012. Rapport d'étude GRETIA pour Ouest Am'. 12 pp.

HeRBRECHT F, 2014. Hyménoptères aculéates. In Ouest-Am', 2014. Suivis faunistiques et floristiques sur le site de Donges-Est. 2013-2014. Rapport Ouest-Am' pour le Grand Port Maritime Nantes Saint-Nazaire / DTAE-Service Environnement. pp. 23 - 36.

Herbrecht F, Aubert M, DUfrêne E, Durand O, Garrin M, Genoud D \& MahÉ G, 2019. Abeilles sauvages des Pays de la Loire: exploitation de culots de piégeage. État et stratégie d'amélioration de la connaissance. Rapport GRETIA, CPIE-Anjou et Observatoire des Abeilles pour la DREAL Pays de la Loire, Nantes, $21 \mathrm{pp}$.

https://pollinisateurs.pnaopie.fr/wpcontent/uploads/2020/04/Rapport-abeillessauvages-Gretia-2019.pdf (consultation octobre 2020)

Herbrecht F, Durand O, Karas F \& QUINETTE JP. (coord) 2015. Invertébrés et milieux remarquables des Pays de la Loire. Promenade naturaliste. Groupe d'étude des invertébrés armoricains. Naturalia publications, Turriers, $224 \mathrm{pp}$.

HERBRECHT F \& LAIR X, 2012a. Inventaire préliminaire des Hyménoptères et des Diptères de la Réserve Naturelle Nationale du Lac de GrandLieu. Rapport GRETIA pour la SNPN, Paris, $14 \mathrm{pp}$.

HeRBRECHT F \& LAIR X, 2012b. Inventaires et suivis entomologiques sur la Réserve Naturelle Régionale des Coteaux du Pont-Barré (Beaulieusur-Layon, FR-49). Année 2011. Rapport GRETIA pour la LPO Anjou, Angers, 71 pp.
HerbreCht F \& LAIR X, 2013. Actualisation des connaissances et compléments d'inventaires sur les invertébrés de la Tourbière de Logné (LoireAtlantique). Année 3 (2012) : diptères, hyménoptères, coléoptères, hétéroptères aquatiques, coléoptères carabiques et araignées. Rapport d'étude du GRETIA, Rennes. 64 pp.

https://side.developpement-

durable.gouv.fr/Default/doc/SYRACUSE/342408/ actualisation-des-connaissances-et-complementsd-inventaires-sur-les-invertebres-de-de-la-

tourbiere- (consultation octobre 2020)

LACHAUd A \& MAHÉ G, 2008. Contribution à la connaissance de la diversité des abeilles sauvages de Loire-Atlantique. Rapport Bretagne VivanteSEPNB, Brest, 90 pp.

MAHÉ G, 2009. Les abeilles du genre Colletes (Hymenoptera, Colletidae) en Presqu'île guérandaise (Loire-Atlantique, France). Osmia 3 : 7-11. https://doi.org/10.47446/OSMIA3.4

MAHÉ G, 2015 (coord.). Les bourdons du Massif armoricain. Atlas de la Loire-Atlantique. Penn ar Bed 221: 1-84.

https://pmb.bretagnevivante.org/pmb/uploads/PAB 2015 221.pdf (consultation octobre 2020)

Michez D, RASMONT P, TERZO M \& VEREECKEN NJ, 2019. Abeilles d'Europe. NAP Editions. Verrières-le-Buisson, France, 547 pp.

NiETO A, ROBERTS SPM, KEMP J, RASMONT P, KuHLMANN M, García Criado M, BiesmeiJer JC, Bogusch P, Dathe HH, De La RÚa P, De MeUlemeester $T$, DehoN M, DeWUlf $A$, Ortiz-SÁnchez FJ, Lhomme P, Pauly A, Potts SG, Praz C, QUaRanta M, RadChenko VG, Scheuchl E, SMit J, Straka J, Terzo M, TOMOZII B, Window J \& MICHEZ D, 2014. European Red List of bees. Luxembourg, Publication Office of the European Union, $84 \mathrm{pp}$. https://doi.org/10.2779/77003

Observatoire Des Abeilles, 2018. Apoidea Armoricana, édition 2018. Listes départementales des abeilles de Bretagne, Pays-de-la-Loire et BasseNormandie.

https://oabeilles.net/wp-content/uploa

ds/2018/07/APOIDEA-ARMORICANA-2018-versi

on-finale.pdf (consultation octobre 2020) 
O'CONNOR RS, KUNIN WE, GaRratT MPD, Potts SG, Roy HE, ANDrews C, Jones CM, Peyton JM, Savage J, Harvey MC, Morris RKA, ROBERTS SPM, WRIGHT I, VANBERGEN AJ \& CARVELL C, 2019. Monitoring insect pollinators and flower visitation: The effectiveness and feasibility of different survey methods. Methods in Ecology and Evolution 10(12) : 21292140. https://doi.org/10.1111/2041-210X.13292

Popic TJ, Davila YC \& Wardle GM, 2013. Evaluation of common methods for sampling invertebrate pollinator assemblages: net sampling out-perform pan traps. PLoS One 8(6): e66665. https://doi.org/10.1371/journal.pone.0066665

Ropars L, DAJOZ I \& GeSLIN B, 2018. La diversité des abeilles parisiennes. Osmia 7 :14-19. https://doi.org/10.47446/OSMIA7.3

Westphal C, Bommarco R, Carré G, Lamborn E, Morison N, Petanidou T, Potts SG, ROBERTS SPM, SZENTGYÖRGYI H, TSCHEUlin T, VAISSIÈRE BE, WOYCIECHOWSKI M, BiesmeiJer JC, Kunin WE, SetTele J \& STEFFAN-DEWENTER I, 2008. Measuring bee diversity in different European habitats and biogeographical regions. Ecological Monographs 78(4): 653-671. https://doi.org/10.1890/07-1292.1 